\title{
Research on Key Techniques of the Verification and Testing System for Spacecraft Propulsion
}

\author{
Wang Junwei ${ }^{1}$,a , Zhang Lei ${ }^{1}$, Xiao Qingsheng ${ }^{1}, \mathrm{Li} \mathrm{Gao}^{1}, \mathrm{Han}_{\mathrm{Xiao}}{ }^{1}$ and Bai Bing ${ }^{1}$ \\ ${ }^{1}$ Beijing Institute of Spacecraft Environment Engineering, Beijing, China
}

\begin{abstract}
Electric propulsion is an important technology in the future space missions. The verification and testing system is mainly used for the manufacturers to fully understand the propulsion behavior in the most diverse conditions, and to indentify and eliminate potential failure modes to advance its validity and reliability, explore the applied potential for space missions. The paper presents a new verification and testing system for spacecraft propulsion and the key technologies needed to be developed are studied. The debugging data and research indicate that ultimate pressure of system is better than the $5.0 \times 10^{-6} \mathrm{~Pa}$. Both the cold plate used for xenon pumping and sputtering protective system get an excellent performance in system. The DSMC numerical simulation method is verified that is beneficial to the research of optimization design and plume effect of electric propulsion in future.
\end{abstract}

\section{Introduction}

In order to meet the space missions of high performance, long life spacecraft propulsion in future, electric propulsion technology is getting increasingly attention in many countries. The United States, Japan, Russia, the European space agency are strengthening in electric propulsion technology research and has been successfully applied in many space missions, electric propulsion technology for the task of deep space exploration and satellites in orbit has great application prospect.

Electric propulsion system needs to pass a comprehensive testing to verify their performance level, service life and compatibility with the environment before practical application. Through lots of environmental testing, researchers can completely understand the performance under different environmental conditions to promote the performance of the system parameters and usage, identify and eliminate potential risks, improve the effectiveness and reliability. At present, advanced research institutions and companies abroad have established the ground experiment test system used in all kinds of electric propulsion system [1-3]. Verification and testing technology of electric propulsion will become the important supplement to electric propulsion technology research in the future.

\section{Typical compositions of verification and testing system}

\section{1) Vacuum vessel system}

Typical design of vacuum vessel in verification and testing system for electric propulsion is horizontal structure, which can be designed as the main vessel and auxiliary vessel while a vacuum

\footnotetext{
${ }^{\text {a }}$ Corresponding author : tobenol@126.com
} 
valve connecting between two segments. The main vessel is the main testing environment, so the design needs to consider the sputtering deposition and pollution effect of working medium. The length design of the main vessel generally should be 4 to 5 times of the radius. Furthermore, taking into account the sputtering effect of main vessel material and magnetic shielding, non-magnetic stainless steel is selected for the vessel.

2) Vacuum pumping system.

At present, the electric propulsion verification and testing system generally adopts the four-stage vacuum pumping configuration to exhaust gas. The typical vacuum system configuration includes the four-stage oil free pumps, the first stage is the rough vacuum system, and the main equipment is the Roots units. The second stage vacuum pumping system is the molecular pump pumping system. The third stage is the high vacuum pumping system, the main equipment includes the cryogenic pump and the fourth stage is the xenon pumping system, which is generally used to remove the working medium of xenon.

3) Temperature control system.

The temperature control system of the electric propulsion verification and testing system consists of the shroud and temperature control unit, wherein the shroud material is made of stainless steel. As shown in figure 1, the structure of shroud is in the diamond honeycomb shape, in which the bulge surface is oriented toward the test piece and the back plate faces the wall of the vessel. Using this kind of shroud can achieve better temperature uniformity.

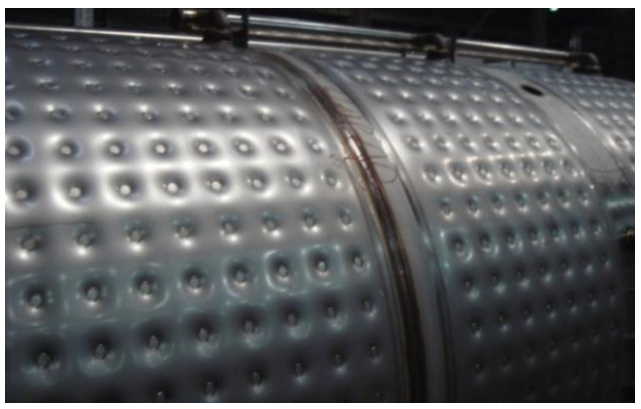

Figure 1. Diamond honeycomb shape shroud using in testing system of electric propulsion

Heat transfer oil refrigeration and heating system can quickly achieve heating and cooling of shroud in the temperature range of $-75^{\circ} \mathrm{C}$ to $+100^{\circ} \mathrm{C}$. Temperature control system adopts watercooling two-stage refrigeration technology, which provides the system refrigeration through the efficient plate heat exchanger and heat source through the electric heating device for the system.

4) Motion platform system

In order to realize the movement of electric propulsion inside the testing vessel, the motion platform system shown in figure 2 is equipped in general. The system design considers the usage under the high and low temperature environment, so the selection of components and structural materials of the platform should meet the harsh conditions of high vacuum and temperature change. In order to meet the requirements of high vacuum environment, the oil parts such as bearings is cleaned and treated with $\mathrm{MoS}_{2}$ solid lubrication.

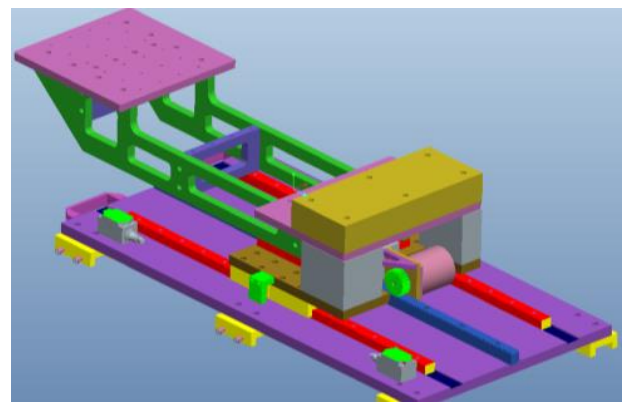


Figure 2. Motion platform system using in testing system of electric propulsion

5) Sputtering protection system

The high-energy particles produced by electric propulsion can produce sputtering corrosion on the structure and materials of testing system when it's working, especially for the high-power and longlife propulsion systems. The sputtering effect will have an impact on the test results, and on the other hand, it also pollutes the thruster itself. Typical sputtering protection system can implement sputtering protection of the whole testing system, which is generally realized through the style of beam target. The beam target adopts the materials with low sputtering rate and achieves full system protection through special incident angle design.

\section{Key technologies of the system}

\subsection{The pumping technology of working medium}

The verification and testing of spacecraft propulsion has its particularity. On the one hand, electric propulsion must be injected the working medium continuously, and the gas medium is transformed into high-energy beam in the vacuum vessel after ionization. Especially for the high power propulsion system, the higher power means that it will consume more gas medium. So the testing system needs to have greater extraction capacity than traditional thermal vacuum testing equipment. On the other hand, the testing should be specially design in vacuum system because of the particularities of xenon. The traditional vacuum pump can't be fully qualified for the extraction of xenon: the molecular pump is relatively small and expensive to the pumping system. The working principle of the diffusion pump may cause oil pollution to the propulsion system. The Cryopump is main pump of the traditional thermal vacuum test, but its pumping capacity for xenon is weak. Due to the higher ion energy of electric propulsion in working, the Cryopump using in electrical propulsion experiment as main pump has many limitations.

In our testing system, the vacuum system adopts cold plate driven by cold head to pumping working gas, and the testing data shows that the method can well meet the needs of ground verification. The cold plates installed in the vessel are shown in figure 3. In theory, the maximum pumping speed on the surface of cryogenic cold plate can be expressed by the following formula:

$$
S_{\max }=\frac{1}{4} \bar{V}_{1} A=\left(\frac{R T_{g}}{Z w M}\right)^{1 / 2} A=3.638 A\left(\frac{T_{g}}{M}\right)^{1 / 2}
$$

Where $A$ is the cold plate area, $\mathrm{cm}^{2} ; T_{g}$ is the temperature of the pumping gas, $\mathrm{K} ; M$ is the molar mass of the gas molecule; $R$ is the gas constant. In fact, due to the influence of gas pressure and condensation coefficient, the actual pumping speed of the cold plate is:

$$
\begin{aligned}
& S_{\alpha}=\alpha S_{\max }\left[1-\frac{P_{s}}{P_{g}}\left(\frac{T_{g}}{T_{s}}\right)^{1 / 2}\right] \\
& =\alpha 3.638 A\left(\frac{T_{g}}{M}\right)^{1 / 2}\left[1-\frac{P_{s}}{P_{g}}\left(\frac{T_{g}}{T_{s}}\right)^{1 / 2}\right]
\end{aligned}
$$

In which $P_{g}$ is the gas pressure, $\mathrm{Pa} ; P_{s}$ is the vapor pressure, $\mathrm{Pa} ; T_{s}$ is cold plate temperature, $\mathrm{K} ; \alpha$ is the condensation coefficient. For the calculation of gas characteristics of xenon, set $P_{g}>>P_{s}, \alpha=1$, and the pumping speed of the cold plate is as follows:

$$
S_{\alpha, X e}=3.638 \sqrt{T g / M}=5.44 \mathrm{~L} / \mathrm{s} \cdot \mathrm{cm}^{2}
$$

The pumping ability of cold plate is proportional to its area. Therefore, in the process of vacuum system configuration, we can calculate the need pumping speed according to the requirement of electric propulsion system such as working flow and working pressure, calculate and equip with enough area of cold plate. 
In the design process of the cold plate, it is also necessary to consider the influence of the system thermal load to the final cooling power required in the system. The thermal load can be calculated by the following formula:

$$
\mathrm{Q}_{\mathrm{th}} \approx \sigma \varepsilon_{r}\left(T_{2}^{4}-T_{1}^{4}\right) A_{1}
$$

In which, $\sigma=5.67 \times 10^{-12} \mathrm{~W} / \mathrm{cm}^{2} K^{4}, \varepsilon_{r}$ is the emissivity of cold plate, $T_{2}, T_{1}$ is the ambient temperature and the cold plate temperature, and $\mathrm{A}_{1}$ is the cold plate area.

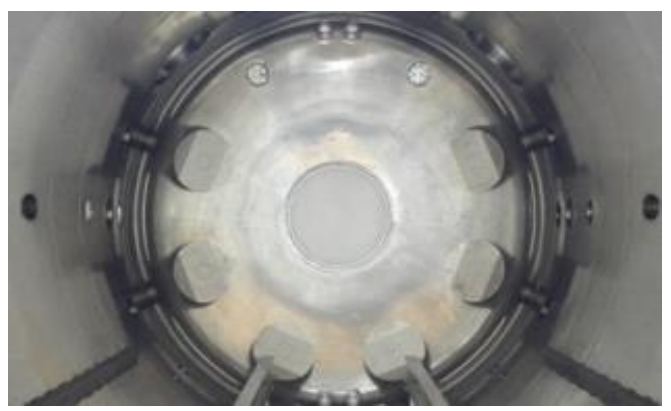

Figure 3. Cold plate driven by cold head in pumping system of the testing vessel

Therefore, in order to guarantee the normal working of the cold plate, the refrigeration power and the cold plate area required by the system has a direct relationship with the environment temperature. The pumping speed is proportional to the area of cold plate, but at the same time the cold plate area is limited by the heat load and the refrigeration power. Thus to improve the pumping ability, the effort should be on the minimization of heat load or selection of high-power refrigeration system of coolingplate.

To reduce the heat load, it is recommended that the shroud and insulation materials are installed in the vessel while the shroud can be cooled by liquid nitrogen. On the one hand, liquid nitrogen shroud can pump part of gas, on the other hand, the shroud greatly reduces the system radiation to cold plate, which improving the abilities of pumping speed of cold plate and running time[4-5].

\subsection{Sputtering protection research}

In order to meet the requirement of long life testing to electric propulsion, the sputtering protection system should be added in system to realize the protection of xenon particles in the ignition process. The design of sputtering protection system is various and pivotal [6-7]. The figure 4 is kinds of protection system using in the testing system abroad.
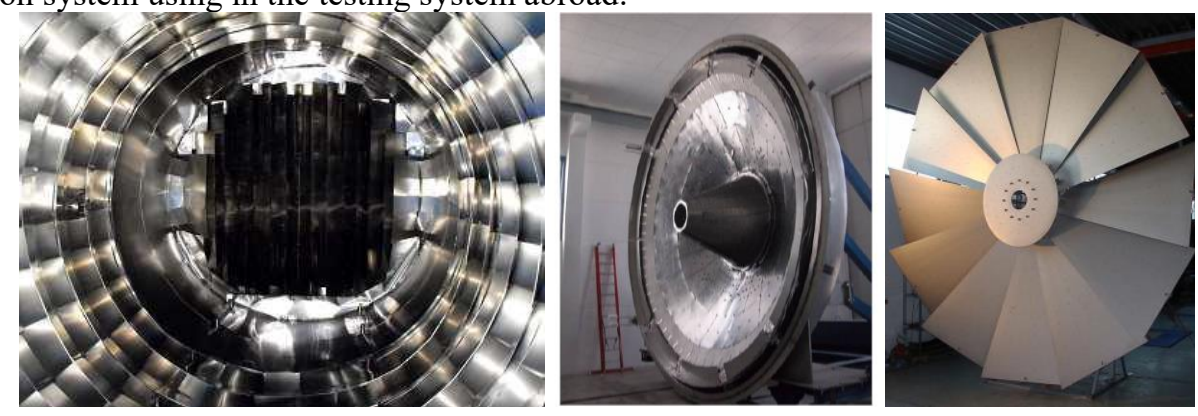

Figure 4. The protection system using in the testing system abroad

The sputtering protection system in our system adopts the similar structure with shroud, which is titanium structure or graphite coated material. In general, sputtering protection system is divided into xenon pump protection unit, the main vessel protection unit and the beam dump installed in front of thruster. The beam dump adopts the structure of the louver while the vessel protection unit is fish 
skeleton structure. For the convenience of subsequent maintenance, the inside of vessel protection unit is detachable type titanium plate protection layer and the outer layer is the welded titanium plate. The structure of xenon pump protection unit is the same with beam dump. The overall structure is shown in figure 5 and the structure of beam dump is shown in figure 6.

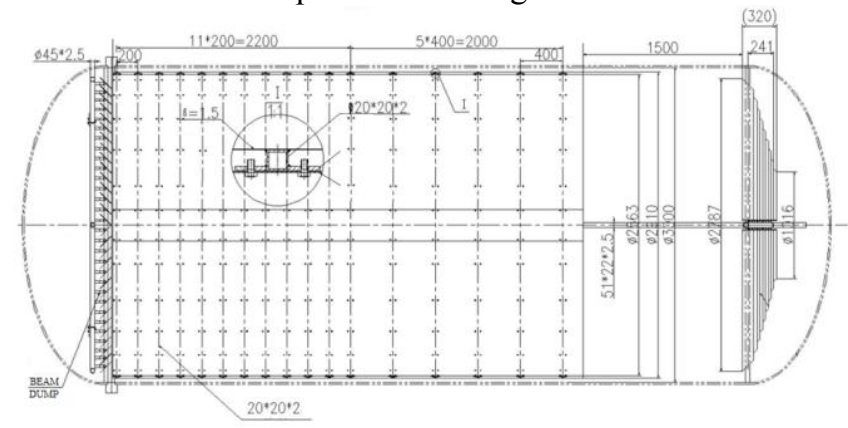

Figure 5. The overall structure of sputtering protection system

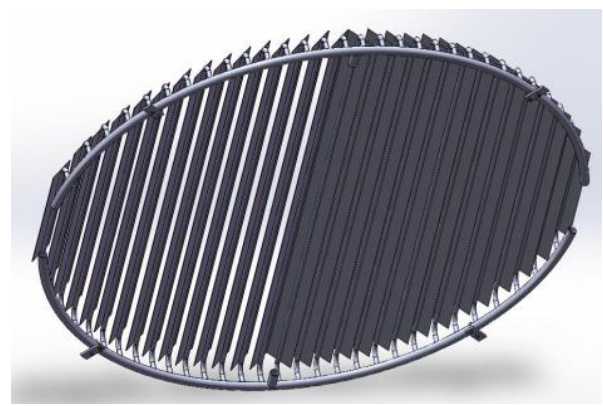

Figure 6. The beam dump installed in front of the thruster

\subsection{System optimization based on DSMC method}

Due to the complex pressure and temperature flow field in the system, the layout of the cold plate and other equipment is essential. The distribution of internal pressure flow field is related to the area, position, structure form of cold plate and sputtering protection system. Due to the difficult by traditional calculation method to carry on the design, it is recommended that the direct Monte Carlo (DSMC) method is used to simulate the vacuum flow field to achieve the optimal design of complete testing system.

The core of DSMC method is using a small amount of simulating molecules instead of the number of gas molecules to simulate the actual physical process. In the simulation, the calculating system tracks simulated molecular, records the change of their position and velocity, calculates the collision between the molecules and the molecules with surface. Through statistical average, various flow parameters can be obtained finally. It can better guide the configuration optimization of electric propulsion testing vacuum system through the numerical simulation of DSMC, which has been applied in advanced research institutions abroad [8-9].

All particles collide with the wall transfer into the neutral particles, then reflect them according to Maxwell's model.In addition to the xenon cold plate and the thruster wall are the exit of particle, the particle can't escape from the other boundary surface. The basic input parameter of simulation is shown in table 1.

Table 1. Basic input parameter of thrusters

\begin{tabular}{|c|c|}
\hline Outside diameter of EP & $100 \mathrm{~mm}$ \\
\hline Inner diameter of EP & $60 \mathrm{~mm}$ \\
\hline
\end{tabular}




\begin{tabular}{|c|c|}
\hline Gas flow & $160 \mathrm{sccm}$ \\
\hline Divergence angle & $45^{\circ}$ \\
\hline Ion velocity of $\mathrm{Xe}^{+}$ & $18000 \mathrm{~m} / \mathrm{s}$ \\
\hline Ion velocity of $\mathrm{Xe}^{++}$ & $25456 \mathrm{~m} / \mathrm{s}$ \\
\hline Atomic temperature of Xe & $273 \mathrm{~K}$ \\
\hline Ion temperature of Xe & $4 \mathrm{eV}$ \\
\hline Wall temperature & $300 \mathrm{~K}$ \\
\hline $\begin{array}{c}\text { Reference temperature of } \\
\text { isothermal model, } \mathrm{T}_{\text {ref }}\end{array}$ & $3.5 \mathrm{eV}$ \\
\hline Reference electron density & $1.0 \mathrm{e}^{16} / \mathrm{m}^{3}$ \\
\hline
\end{tabular}

The background pressure in the vessel is the main factor which affect the measurement data of electric propulsion. The internal pressure of the vessel is made up of $\mathrm{Xe}$ atoms, $\mathrm{Xe}^{+}$ion and $\mathrm{Xe}^{++}$ion density. Due to the slower velocity of Xe atomic, the accumulation effect is obvious. At the same time, due to collision with wall, Xe atomic number is higher than that of Xe ions, which also cause the Xe atomic is the main part of the pressure distribution.

The simulation is shown in the figure 7, (a) is the original schemes without any sputtering protection devices. the position of the cold plate in (b) and (c) is the same, and the different is that a circular protection baffle is arranged in front of the cold plate in the latter section of the vessel in (c). By contrast, it can be seen that the blocking effect of the circular protection baffle structure on the reflux particles is obvious, and lots of particles are adsorbed by the pieces of xenon cold plates set in the latter section of the vessel, which reduces the overall pressure of the latter section. However, the circular protection baffle has no obvious effect on the particles in front part of vessel, so the pressure distribution in the front of the vessel is similar in the two optimization schemes.

The beam target is setted up in the end head of the testing system in Figure (d), The particle enteres the back head of vessel after collision with beam dump, which leads the particle's accumulation and increasement of pressure in end head, which illustrates that the $\mathrm{Xe}^{+}$ions with higher speed and CEX particle collide with beam dump and lose its charge. The motion speed dropped to level of thermal motion and particles accumulates in this aera. The pressure in front of baffle is lower than other schemes, so the scheme can provide a better ignition environment in the place of thrusters. It also can be seen from the diagram that the xenon cold plate should be added to reduce the pressure in the end head.

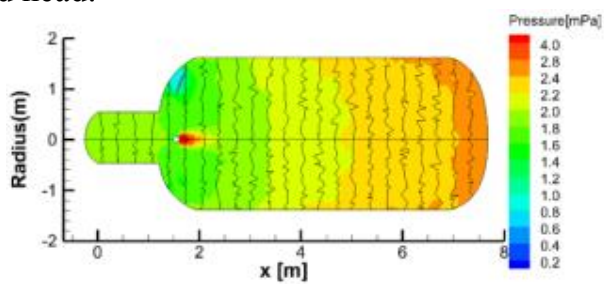

(a)

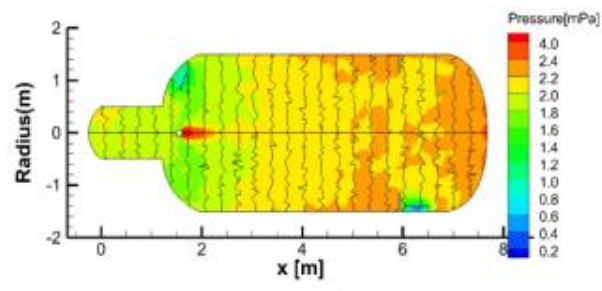

(c)

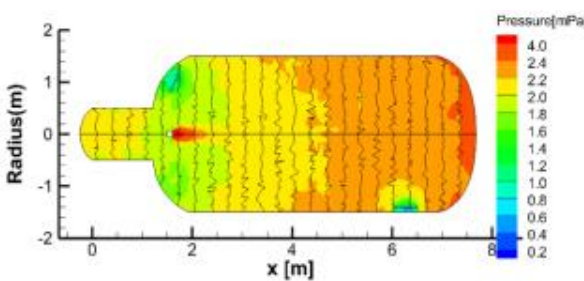

(b)

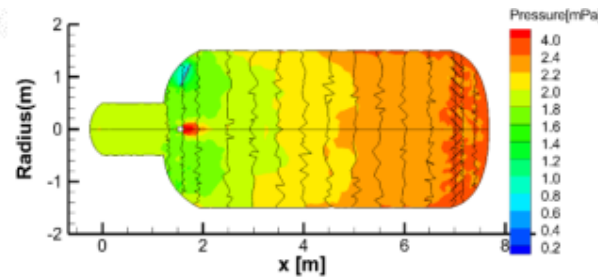

(d)

Figure 7. The simulation and optimization design of testing system 


\section{Conclusions}

Based on the research of the key technology, the final validation and testing system for spacecraft electric propulsion get an excellent performance, and the ultimate pressure of vacuum system is better than the $5.0 \times 10^{-6} \mathrm{~Pa}$. The pumping capacity of cold plate can satisfy the demand of high-power electric propulsion using xenon gas while the sputtering protective system has played a certain protective effect to all system. Through the DSMC numerical simulation, it indicates that the distribution of pressure field is related to area, position, structure of cold plate and sputtering protective system. The numerical simulation method can better guide the configuration optimization of electric propulsion testing system. The method has been applied in advanced research institutions abroad and proved that it's beneficial to the research to the optimization design of vacuum system and plume effect research of electric propulsion.

\section{References}

1. Neumann A, Holz A, Dettleff G, et al. The new DLR high vacuum test facility STG-ET[C]. The 32nd International Electric Propulsion Conference, Wiesbaden, Germany. 2011:1-10

2. M. Saverdi, M. Signori, L. Milaneschi, et al. The IV10 Space Simulator for High Power Electric Propulsion Testing: Performance Improvements and Operation Status[C]. The 30th International Electric Propulsion Conference, 2007:1-13

3. M. Andrenucci, M. Berti, L. Biagioni, et al. The New EP Test Facilities at Centrospazio and Alta[C]. The 28th International Electric Propulsion Conference, 2003:1-9

4. Andrew D. Ketsdever. Design Considerations for Cryogenic Pumping Arrays in SpacecraftThruster Interaction Facilities [J]. Journal Of Spacecraft And Rockets Vol. 38, No. 3, May-June 2001

5. Leonardo Biagioni, Andrea Passaro, Mariano Andrenucci. Particle Simulation of Tailored Vacuum Pumping Configurations for Electric Propulsion Testing[C]. Fourth International Symposium Environmental Testing for Space Programmes, ESA SP-467, 2001

6. Passaro A, Vicini A, Biagioni L. Plasma Thruster Plume Simulation: Effect of Vacuum Chamber Environment[C]. The 35th AIAA Plasmadynamics and Lasers Conference 28 June-1 July 2004, Portland, Oregon

7. Andrew D. Ketsdever. An Overview of Ground Based Spacecraft-Thruster Interactions Studies: Facility Design Issues[C]. The 38th Aerospace Sciences Meeting \& Exhibit. AIAA 2000-0463

8. Biagioni L, Kim V, Nicolini D, et al. Basic issues in electric propulsion testing and the need for international standards[C]. The 28th International Electric Propulsion Conference. Toulouse, France. IEPC Paper. 2003, 230: 17-21

9. L. Biagioni, L. Boccaletto, S. Marcuccio, D. A Large Space Simulator for Electric Propulsion Testing: Design Requirements and Engineering Analysis[C]. The 36th AIAA/ASME/SAE/ASEE Joint Propulsion Conference. AIAA 2000-3750 